\title{
ACCRETION OF PROTOBINARY AND EVOLUTION OF THE MASS RATIO
}

\author{
Tomoyuki Hanawa, ${ }^{1,2}$ Yasuhiro Ochi, ${ }^{1}$ and Kanako Sugimoto ${ }^{1}$
}

\begin{abstract}
RESUMEN
Reexaminamos la acreción en un sistema protobinario mediante simulaciones numéricas en dos dimensiones. Consideramos estrellas en órbitas circulares en torno al centro de masa. Suponemos que el flujo de acreción del gas ocurre en el plano orbital. El gas se inyecta desde un círculo con un radio 5 veces mayor que el de la separación orbital. El gas inyectado tiene densidad superficial, velocidad de caída y momento angular específico constantes. La acreción depende del momento angular específico del gas inyectado, $j_{\text {inf }}$. Cuando $j_{\text {inf }}$ es pequeño, la binaria acrece gas principalmente por dos canales: los puntos de Lagrange $\mathrm{L}_{2}$ y $\mathrm{L}_{3}$. Cuando $j_{\text {inf }}$ es grande, la binaria acrece gas sólo por el punto $\mathrm{L}_{2}$. En ambos casos, la primaria acrece más que la secundaria pese a que el punto $\mathrm{L}_{2}$ está más cerca de la secundaria. Después de pasar por el punto $\mathrm{L}_{2}$, el gas fluye alrededor de la secundaria y a través del punto $\mathrm{L}_{1}$ hacia la primaria. Sólo una pequeña fracción del gas regresa a la secundaria; el restante forma un anillo circunestelar alrededor de la primaria. La acreción hace que el cociente de masa $q=M_{2} / M_{1}$, decrezca, donde $M_{1}$ y $M_{2}{ }^{* * * * * * *}$. La tasa de acreción aumenta con el tiempo. Para $j_{\text {inf }}$ grandes, es despreciable durante los primeros períodos de rotación.
\end{abstract}

\begin{abstract}
We have reexamined accretion in a protobinary system with two dimensional numerical simulations. We consider protostars which rotate around the center of the mass with circular orbits. The accreting gas is assumed to flow in the orbital plane. It is injected from a circle whose radius is 5 times larger than the orbital separation of the binary. The injected gas has constant surface density, infall velocity, and specific angular momentum. The accretion depends on the specific angular momentum of the injected gas, $j_{\text {inf }}$. When $j_{\text {inf }}$ is small, the binary accretes the gas mainly through two channels: one through the Lagrangian point $\mathrm{L}_{2}$ and the other through $\mathrm{L}_{3}$. When $j_{\text {inf }}$ is large, the binary accretes the gas only through the $\mathrm{L}_{2}$ point. The primary accretes more than the secondary in both cases, although the $\mathrm{L}_{2}$ point is closer to the secondary. After flowing through the $\mathrm{L}_{2}$ point, the gas flows half around the secondary and through the $\mathrm{L}_{1}$ point to the primary. Only a small amount of gas flows back to the secondary and the rest forms a circumstellar ring around the primary. The accretion decreases the mass ratio, $q=M_{2} / M_{1}$, where $M_{1}$ and $M_{2}$ denote the masses of the primary and secondary, respectively. The accretion rate increases with time. When $j_{\text {inf }}$ is large, it is negligibly small in the first few rotation periods.
\end{abstract}

\section{Key Words: ACCRETION, ACCRETICN DISCS - BINARIES: GENERAL - HYDRODYNAMICS - STARS: PRE-MAIN SEQUENCE}

\section{INTRODUCTION}

The masses of stars are acquired mostily through accretion during their protostellar phase. The accretion is most likely through a circumstellar disk since each component star has an accretion disk in most young binary systems. The accretion disk is most likely to be replenished by accretion from a circumbinary disk. In fact, circumbinary disks are observed in some young binary systems as molecular rings (see, e.g., Close et al. 1998). If gas is accreted from the circumbinary disk to the circumstellar disks, the accretion rates will be nearly equal

\footnotetext{
${ }^{1}$ Department of Astrophysics, Nagoya University, Japan.

${ }^{2}$ Present address: Center for Frontier Science, Chiba University, Japan.
}

to the accretion rates of the primary and secondary stars in the long term. In other words, the accretion from the circumbinary disk controls the accretion of each component star.

The accretion in a protobinary system has been studied by Bate \& Bonnell (1997b) with hydrodynamical simulations. In their simulations the secondary accretes more gas than the primary when the accreting gas has a moderately large specific angular momentum. The result is also consistent with the earlier ballistic models (Artymowicz 1983, Bate \& Bonnell 1997a), and was explained by the fact that the secondary has a larger orbit than the primary.

We have reexamined the accretion and have obtained the opposite result that the primary accretes 
more than the secondary. When the gas has a moderately large angular momentum, the gas flows into the binary system through the $\mathrm{L}_{2}$ point, the Lagrange point close to the secondary. It flows, however, and does not fall directly onto the secondary. It flows half around the secondary from the $\mathrm{L}_{2}$ point to the $\mathrm{L}_{1}$ point, where $L_{1}$ denotes the Lagrange point between the primary and secondary. Then it flows around the primary and forms a circumstellar ring. Only a small amount of the gas flows back to the secondary through the $\mathrm{L}_{1}$ point. Consequently the secondary has a less massive circumstellar ring.

This paper is organized as follows. Our model and numerical methods are given in $\S 2$. Numerical results are shown in $\S 3$. A brief summary is given in $\S 4$.

\section{MODEL AND METHOD OF COMPUTATION}

We consider two-dimensional flow accreting onto a binary. The accreting gas is assumed to be isothermal and the sound speed, $c_{\mathrm{s}}$, is constant. The self gravity of the accreting gas is ignored for simplicity. We also ignore the increase in the mass of stars through accretion for simplicity. Thus the masses of the primary and secondary stars are set constant at $M_{1}$ and $M_{2}$, respectively. The primary and secondary stars are assumed to have circular orbits. $M=M_{1}+M_{2}$, and the mass ratio, $q=M_{2} / M_{1}$. The barycenter of the system is assumed to coincide with the origin of the Cartesian coordinates.

The hydrodynamical equations are solved on a Cartesian grid with a second order accurate finite difference scheme. Our difference scheme is based on the TVD scheme of Roe (1981). We achieved the second order accuracy using MUSCL (see, e.g., Hirsch 1990).

The Cartesian grid covers the square region of $-L \leq x, y \leq L$ and the value of $L$ is taken to be $5.12 a$ in most computations. The Cartesian grid contains $1024 \times 1024$ square cells in most models and $1536 \times 1536$ square cells in high resolution models. The center of the grid coincides with the barycenter.

At the initial stage $(t=0)$, the surface density is set to be

$$
\Sigma=\left\{\begin{array}{ll}
1 & \sqrt{x^{2}+y^{2}} \geq R_{\text {out }} \\
0.01 & \sqrt{x^{2}+y^{2}}<R_{\text {out }}
\end{array},\right.
$$

where $R_{\text {out }}$ denotes the radius of the effective outer boundary. The initial velocity is set to be

$$
\boldsymbol{v}_{0}=\frac{j_{\text {inf }}}{R_{\mathrm{out}}^{2}}\left(\begin{array}{c}
-y \\
x
\end{array}\right)-\frac{v_{\mathrm{inf}}}{\sqrt{x^{2}+y^{2}}}\left(\begin{array}{l}
x \\
y
\end{array}\right),
$$

where $v_{\text {inf }}$ and $j_{\text {inf }}$ are model parameters specifying the radial infall velocity and the specific angular momentum of the infalling gas, respectively. The surface density and velocity are kept at the initial values in the region of $r \geq R_{\text {out }}$ Thus we have a constant inflow from the boundary of $r=R_{\text {out }}$ at the rate of $\dot{M}=2 \pi R_{\text {out }} v_{\text {inf }}$.

The infall velocity, $v_{\text {inf }}$, is given so that the energy of the infalling is vanishingly small at the outer boundary.

We show our numerical simulations in the nondimensional form. For comparison with Bate \& Bonnell (1997b) we use the same unit. Namely the separation $a$ is used as the unit length and the angular velocity, $\omega$, is taken to be unit frequency. Accordingly the total mass of the binary is $G M=1$. In most models we compute accretion for 15 rotation periods, i.e., from $t=0$ to $30 \pi$.

\section{RESULTS}

$$
\text { 3.1. } q=0.6 \text { and } j_{\text {inf }}=1.6
$$

As a typical example we show model 6-16 in which the parameters are set to be $q=0.6$ and $j_{\text {inf }}=1.6$. The sound speed is $c_{\mathrm{s}}=0.25$ and the flow is mildly cold.

The infalling gas forms a ring around the binary at $t \simeq 2 \pi$. The gas flows inward at a supersonic speed outside the ring while it flows outward inside the ring. The ring is the shock front formed by the infalling gas. Its inner radius coincides with the centrifugal barrier, at which the radial velocity reverses owing to the increase in the rotation velocity.

The upper panel of Figure 1 denotes the surface density and velocity at $t=4.0 \pi$ by the grayness and arrows, respectively. The contours denote the Roche potential. The ring has two arms, one of which extends toward the Lagrange point, $\mathrm{L}_{2}$. The arm continues to a semicircle bridging the Lagrange points, $\mathrm{L}_{2}$ and $\mathrm{L}_{1}$. Gas is supplied through the semicircle to the inner ring surrounding the primary star. The other arm extends toward the Lagrange point, $\mathrm{L}_{3}$, but is not linked with the inner ring. The circumprimary ring is more massive than the circumsecondary one.

The lower panel of Figure 1 is the same as the upper one but for $t=16.0 \pi$. The inner and outer rings are thicker than those at $t=4.0 \pi$. Gas is supplied to the inner rings also through the Lagrange point, $\mathrm{L}_{3}$. The gas supply is minor and much smaller than that through $\mathrm{L}_{2}$.

We look at the stage of $t=8.0 \pi$ in Figure 2 . The arrows denote the velocity in the corotation frame, while the grayness denotes the surface density. The flow is nearly stationary in the corotation 

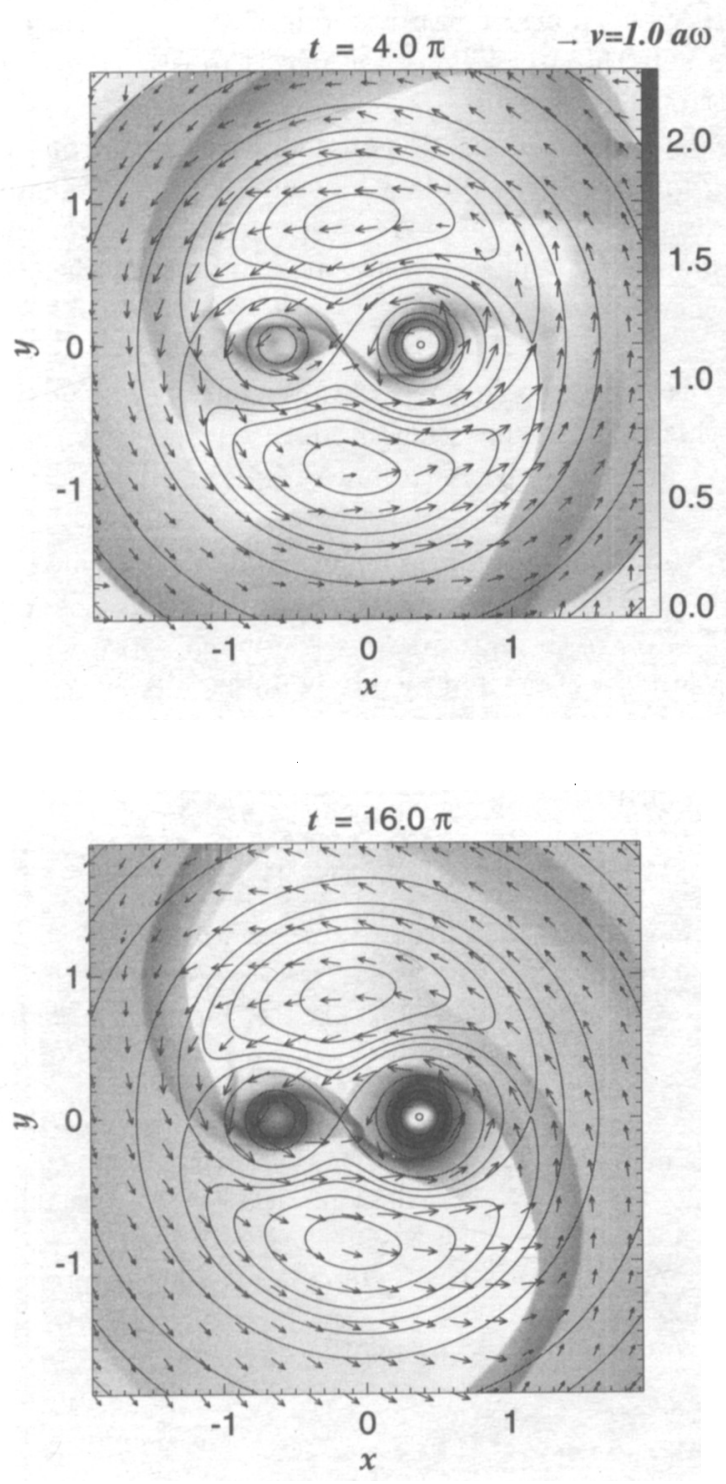

Fig. 1. Evolution of the surface density (grayness) and velocity (arrows) in model 6-16 in which the model parameters are set to be $q=0.6, j_{\text {inf }}=1.6$, and $c_{\mathrm{s}}=0.25$. The upper panel denotes the stage of $t=4.0 \pi$ while the lower panel does that of $t=16.0 \pi$.

frame. It is along the equipotential surface near the binary. The circumbinary ring extends in the region of $1.5 \lesssim r \lesssim 4$. The circumbinary ring is inhomogeneous while the average surface density is $\Sigma \sim 20$. The spiral arms are density waves in the circumbinary ring (Artymowicz \& Lubow 1996). The gas flows not along but across the spiral arms. The spiral arms extract the angular momentum for the gas to accrete onto the binary.

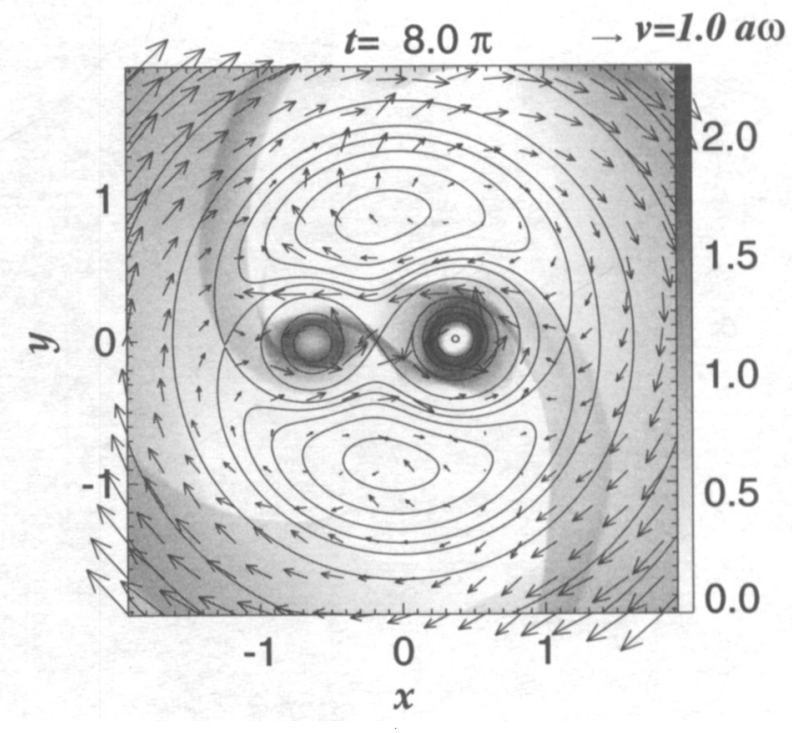

Fig. 2. The same as Figure 1 but for $t=8.0 \pi$. The arrows denote the velocity measured in the corotation frame.

Figure 3 denotes the mass of the circumstellar disks as a function of time. We define the circumprimary disk to be the gas enclosed in the circle whose center is located at the primary and which passes through $\mathrm{L}_{1}$. The circumsecondary disk is defined similarly. The mass of the circumprimary disk, $M_{1 \mathrm{~d}}$, denoted by the solid curve is larger than that of the circumsecondary disk, $M_{2 \mathrm{~d}}$, denoted by the dash-dotted curve. The dashed curve denotes $M_{\mathrm{d}} \equiv M_{1 \mathrm{~d}}+M_{2 \mathrm{~d}}$. The mass ratio decreases since $M_{1 \mathrm{~d}}>M_{2 \mathrm{~d}} / q$. The latter is denoted by the dotted curve in Figure 3.

\subsection{Dependence on $j_{\text {inf }}$}

We have made models of $j_{\text {inf }}=1.3,1.4,1.5,1.7$, 1.8 , and 1.9 for $q=0.6$ and $c_{\mathrm{s}}=0.25$ to study dependence on $j_{\text {inf }}$. When $j_{\text {inf }}$ is smaller, the binary accretes gas at a higher rate. When $j_{\text {inf }} \leq 1.4$, the gas accretes into the binary not only through the $\mathrm{L}_{2}$ point but through the $\mathrm{L}_{3}$ point. When $j_{\text {inf }} \geq 1.8$, the accretion rate is negligibly small during the first few rotation periods. The period of negligibly small accretion rate is longer for a larger $j_{\text {inf }}$.

\subsection{Dependence on $c_{\mathrm{s}}$}

We have made models of $c_{\mathrm{s}}=0.20$ and 0.30 for $j_{\text {inf }}=1.9$ and $q=0.6$. The period of negligibly small accretion rate is longer for a smaller $c_{\mathrm{s}}$. This supports the idea that excess angular momentum is extracted by the shock waves. 


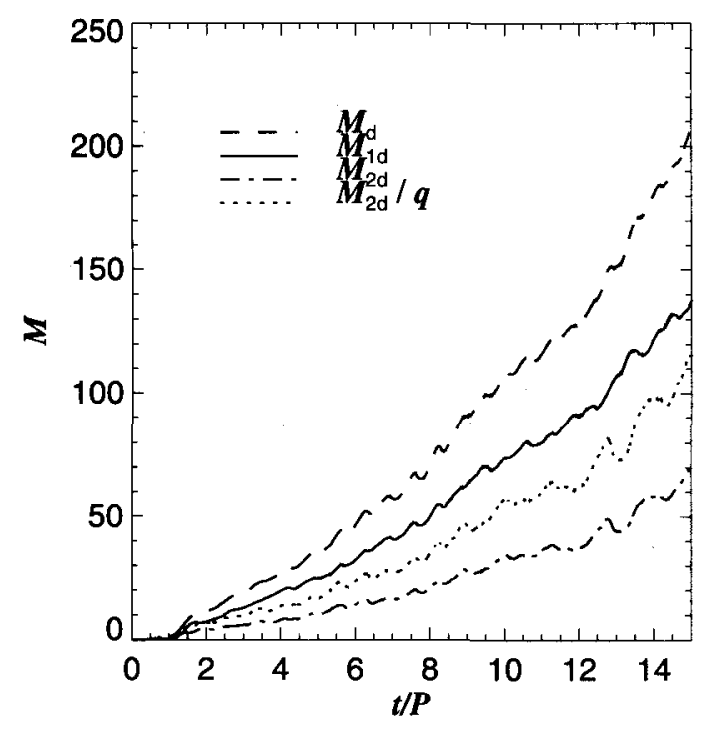

Fig. 3. The masses of the circumprimary and circumsecondary disks are shown as a function of time for model of $q=0.6, j_{\text {inf }}=1.6$, and $c_{\mathrm{s}}=0.25$.

\subsection{Dependence on $q$}

We have made models of $q=0.4,0.8$, and 0.9 for $j_{\text {inf }}=1.6$ and $c_{\mathrm{s}}=0.25$ to study dependence on $q$. The circumprimary disk is more massive than the circumsecondary disk in all the models. Since $M_{1 \mathrm{~d}} \geq M_{2 \mathrm{~d}} / q$, the mass ratio decreases or changes little in all the models.

\section{SUMMARY}

We have obtained a result different from Bate \& Bonnell (1997b). The circumprimary disk is more massive than the circumsecondary one in all the models studied, although the circumsecondary one was more massive for a moderate $j_{\text {inf }}$ in Bate \& Bonnell 1997b). The difference is most likely to be attributed to their large numerical viscosity. As shown in $\S 3$, the gas does not flow directly from the $\mathrm{L}_{2}$ point to the secondary since it has an appreciable angular momentum. We suspect that the angular momentum was lost by the large numerical viscosity in their simulations.

We have shown only a limited number of models in this paper. The other models will be shown later in Ochi, Sugimoto \& Hanawa (2004).

Numerical simulations have been performed on Fujitsu VPP5000 at the Astronomical Data Analysis Center of the National Astronomical Observatory, Japan (myo79c). This study is financially supported in part by the Grant-in-Aid for Scientific Research (C) (13640237) of Japan Society of Promotion of Science (JSPS).

\section{REFERENCES}

Artymowicz, P. 1983, Acta Astronomica, 33, 223

Artymowicz, P., \& Lubow, S. H. 1996, ApJ, 467, L77.

Bate, M. R., \& Bonnell, I. A. 1997a, MNRAS, 285, 16

Bate, M. R., \& Bonnell, I. A. 1997b, MNRAS, 285, 33

Close, L. M., Dutrey, A., Roddier, F., Guilloteau, S., Roddier, C., Northcott, M., Menard, F., Duvert, G., Graves, J. E., \& Potter, D. 1998, ApJ, 499, 883

Hirsch, C. 1990 Numerical Computation of Internal and External Flows, vol. 2 (Chichester: Wiley)

Ochi, Y., Sugimoto, K., \& Hanawa, T. 2004, in preparation

Roe, P. L. 1981, J. Comput. Phys., 43, 357

Tomoyuki Hanawa, Yasuhiro Ochi, and Kanako Sugimoto: Department of Astrophysics, Nagoya University, Chikusa-ku, Nagoya 464-8602, Japan (hanawa,yasuhiro,kana@a.phys.nagoya-u.ac.jp). 


\section{DISCUSSION}

Scarfe - Do your results depend on the sizes of the stars within their lobes, or, put another way, on their surface gravitational potentials?

Hanawa - The results depend little on the assumed radii as long as they are appreciably smaller than the Hill radius.

Farbiash - What happens when the gas gravitational potential is not zero?

Hanawa - We expect several effects. (1) The circumbinary disk may suffer from self-gravitational instability, which enhances the accretion onto the binary. (2) The orbit of the binary may expand due to the disk gravity. (3) The mass of the binary will increase and the orbital elements evolve.

Bate - You have used a very large sound speed in your calculation, and I notice that as you decrease the sound speed $\dot{M}_{2} / \dot{M}_{1}$ increases. Why did you use such a large sound speed? This could be one of the main differences between your results and those of Bate \& Bonnell 1997, apart from differences between 2-D and 3-D.

Hanawa - We assumed a rather high $C_{s}$ for technical reasons. It is difficult to compute the case of low $C_{s}$, i.e., the case of a high Mach number. Although we confirmed that our results depend little on $C_{s}$ in the range studied, the results might differ for a very low $C_{s}$.

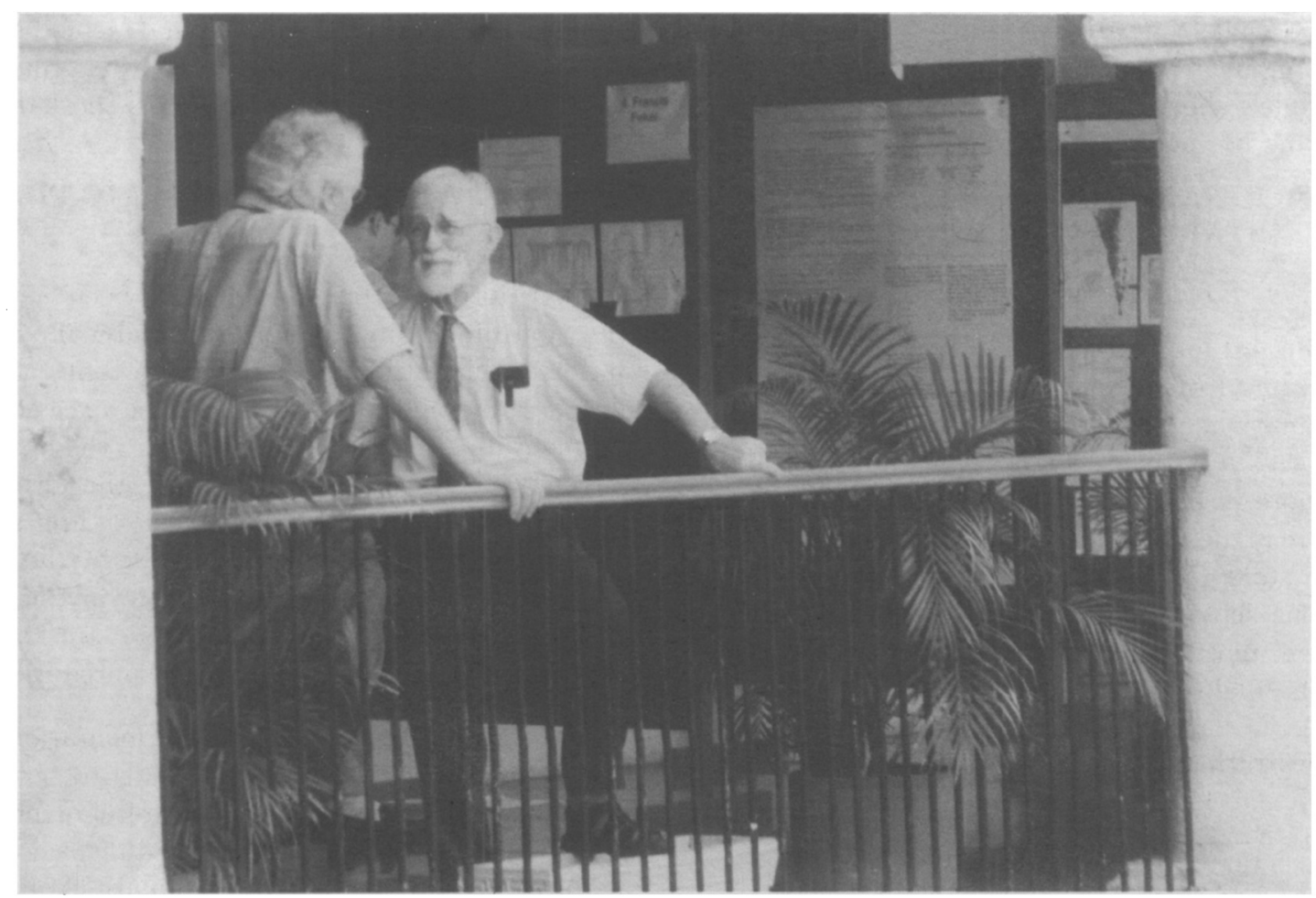

Bo Reipurth and Colin Scarfe. 